\title{
Effect of Core Size on Performance of Fused-Ring Electron Acceptors
}

\author{
Shuixing Dai, ${ }^{\dagger}$ Yiqun Xiao, ${ }^{\ddagger}$ Peiyao Xue, ${ }^{\dagger}$ Jeromy James Rech, ${ }^{\perp}$ Kuan Liu, ${ }^{\dagger}$ Zeyuan Li, ${ }^{\dagger}$ Xinhui Lu, ${ }^{\ddagger}$ \\ Wei You, ${ }^{\perp}$ and Xiaowei Zhan*, ${ }^{*}+$ (C) \\ ${ }^{\dagger}$ Department of Materials Science and Engineering, College of Engineering, Key Laboratory of Polymer Chemistry and Physics of \\ Ministry of Education, Peking University, Beijing 100871, China \\ ${ }^{\ddagger}$ Department of Physics, The Chinese University of Hong Kong, New Territories, Hong Kong, China \\ ${ }^{\perp}$ Department of Chemistry, University of North Carolina at Chapel Hill, Chapel Hill, North Carolina 27599, United States
}

\begin{abstract}
We report 4 fused-ring electron acceptors (FREAs) with the same end-groups and side-chains but different cores, whose sizes range from 5 to 11 fused rings. The core size has considerable effects on the electronic, optical, charge transport, morphological, and photovoltaic properties of the FREAs. Extending the core size leads to red-shift of absorption spectra, upshift of the energy levels, and enhancement of molecular packing and electron mobility. From 5 to 9 fused rings, the core size extension can simultaneously enhance open-circuit voltage $\left(V_{\mathrm{OC}}\right)$, short-circuit current density $\left(J_{\mathrm{SC}}\right)$, and fill factor $(\mathrm{FF})$ of organic solar cells (OSCs). The best efficiency of the binary-blend

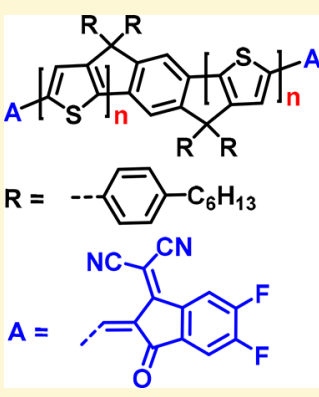
$\begin{array}{ll}n=1 & F 5 I C \\ n=2 & F 7 I C \\ n=3 & F 9 I C \\ n=4 & \text { F11IC }\end{array}$ devices increases from 5.6 to $11.7 \%$, while the best efficiency of the ternary-blend devices increases from 6.3 to $12.6 \%$ as the acceptor core size extends.
\end{abstract}

\section{INTRODUCTION}

Organic solar cells (OSCs) have been proved to be a promising candidate to utilize solar energy, ${ }^{1-3}$ and the power conversion efficiencies (PCEs) have reached over $11 \%$ in fullerene-based OSCs ${ }^{4,5}$ and over $13 \%$ in nonfullerene-based OSCs in recent years. ${ }^{6-9}$ Fullerene acceptors have been the predominant choice in the acceptor materials of OSCs for two decades; however, the limited tunability of electronic properties and weak absorption of fullerene derivatives in visible range hinder further development of OSCs. In contrast, electronic properties of nonfullerene acceptors can be readily tuned by chemical tailoring, providing an effective avenue toward higher performance of OSCs. ${ }^{10-24}$

In 2015, we reported a new class of nonfullerene acceptors, fused-ring electron acceptor (FREA). Generally, FREA consists of an electron-donating fused-ring core and two electronwithdrawing end-groups. ${ }^{25}$ Recently, some high-efficiency FREAs have been reported, ${ }^{25-44}$ among them, different core sizes, such as fused-4-ring, ${ }^{26}$ fused-5-ring, ${ }^{27-29}$ fused-6ring, ${ }^{30-32}$ fused-7-ring, ${ }^{25,33-40}$ fused-8-ring, ${ }^{30,41,42}$ fused-9ring, ${ }^{43}$ fused-10-ring, ${ }^{30,44}$ and fused-11-ring, ${ }^{40}$ have been used individually, but systematic comparisons of different cores have rarely been reported. ${ }^{30}$ These FREAs based on different cores usually contain different end-groups and side-chains, and they are often paired with different donor materials to fabricate devices with different performance. Under this situation, it is impossible to rationally compare these FREAs with different cores or properly understand how the chemical nature of the core and its size would affect performance of the FREAs.
Here, we designed and synthesized a new fused-11-ring core, 7,7,15,15-tetrakis(4-hexylphenyl)-indacenobis(quadra-thienoacene) (IBQT), which was end-capped with 2-(5,6-difluoro-3oxo-2,3-dihydro- $1 \mathrm{H}$-inden-1-ylidene)malononitrile $(2 \mathrm{FIC})^{43}$ to afford a new FREA, F11IC. We then synthesized another new FREA, F5IC, based on fused-5-ring core end-capped with 2FIC. These two new FREAs, together with our previously reported $\mathrm{F}$ IC ${ }^{40}$ and F9IC, ${ }^{43}$ construct a library of structurally closely related FREAs. Specifically, F5IC, F7IC, F9IC, and F11IC (Chart 1) have the same end-groups and side-chains but different core sizes. With this small library, we are able to probe the effects of the core size on electronic, optical, chargetransport, morphological, and photovoltaic properties of the FREAs. We discover that the increase in the core size leads to upshift of energy levels, red-shift of absorption spectra, reduction of bandgaps, and increase in electron mobility of the acceptors. OSCs based on binary blends of these electron acceptors and a large-bandgap polymer donor FTAZ (Chart 1) exhibit PCEs of 5.6-11.7\%; OSCs based on ternary blends of FTAZ/F5IC/F11IC, FTAZ/F7IC/F11IC, and FTAZ/F9IC/ F11IC exhibit PCEs of $6.3-12.6 \%$.

\section{RESULTS AND DISCUSSION}

Synthesis and Characterization. The synthetic routes for F5IC and F11IC are shown in Scheme S1, and the synthesis

Received: May 26, 2018

Revised: July 1, 2018

Published: July 2, 2018 
Chart 1. Chemical Structures of F5IC, F7IC, F9IC, F11IC, and FTAZ

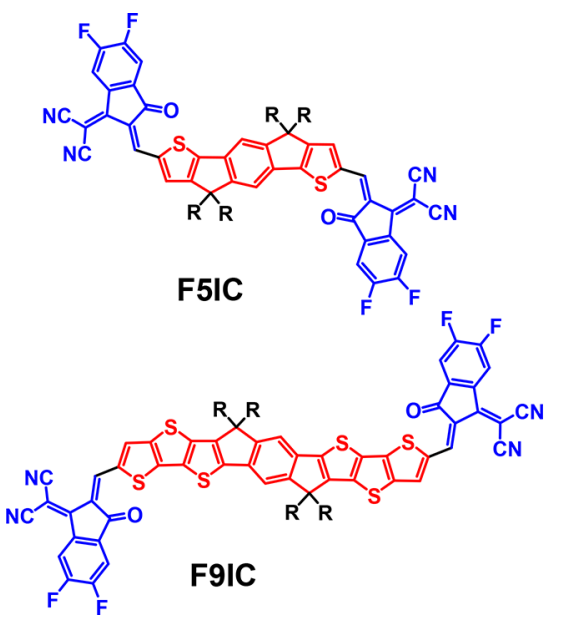

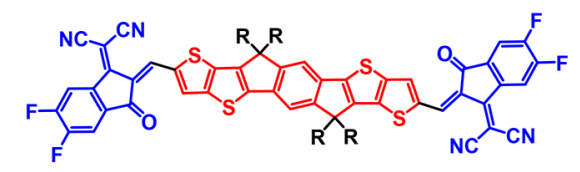

F7IC
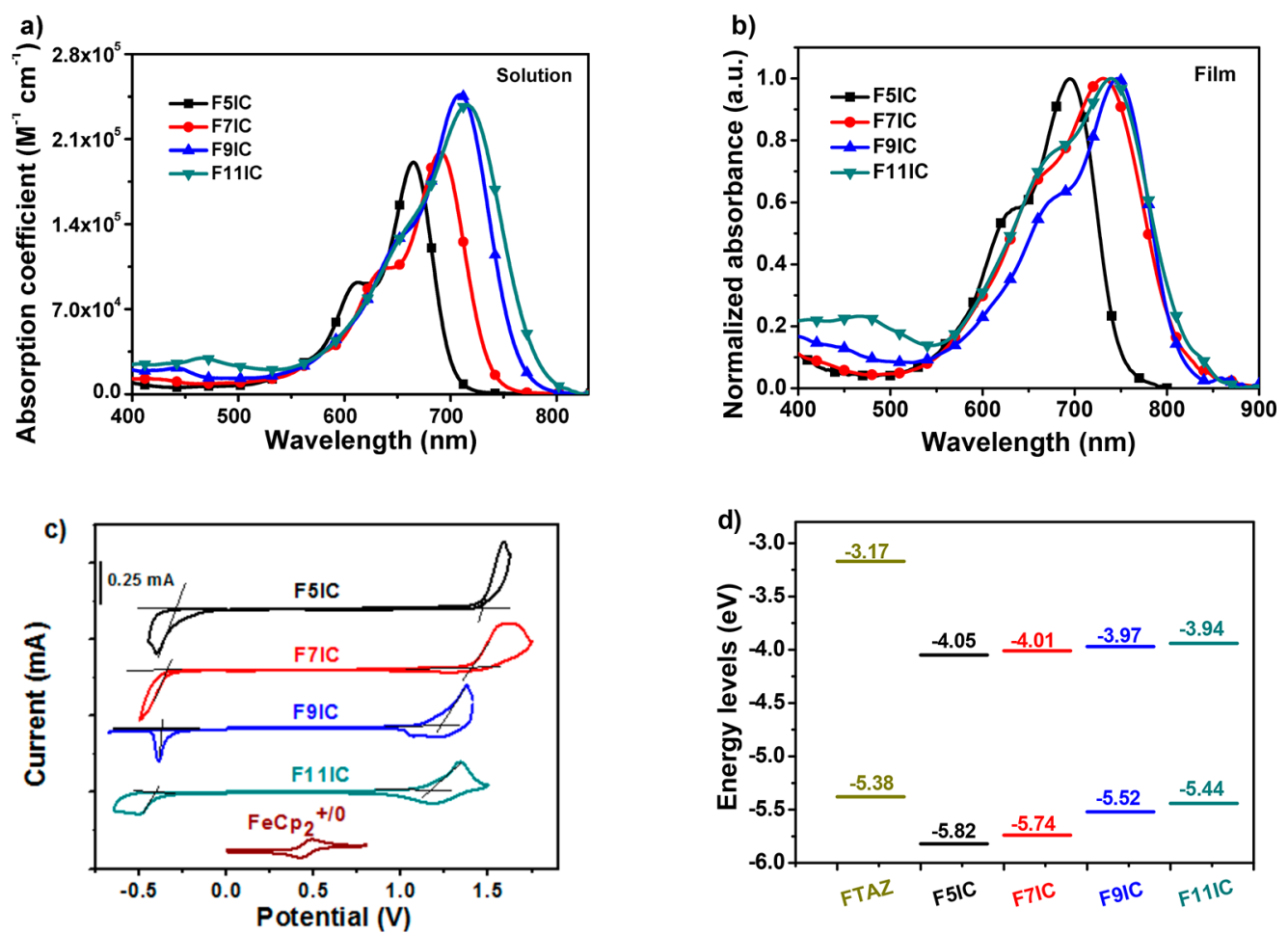

Figure 1. (a) Absorption spectra in chloroform and (b) as a thin film; (c) cyclic voltammograms and (d) energy levels for F5IC, F7IC, F9IC, and F11IC.

\section{Table 1. Absorption and Energy Levels of the Acceptors}

\begin{tabular}{|c|c|c|c|c|c|c|c|c|c|c|}
\hline \multirow[b]{2}{*}{ compound } & \multicolumn{2}{|c|}{$\lambda_{\max }(\mathrm{nm})$} & \multirow[b]{2}{*}{$\varepsilon^{a}\left(\mathrm{M}^{-1} \mathrm{~cm}^{-1}\right)$} & \multirow[b]{2}{*}{$E_{\mathrm{g}}{ }^{\mathrm{opt} b}(\mathrm{eV})$} & \multirow[b]{2}{*}{$E_{\mathrm{ox}}^{c}(\mathrm{~V})$} & \multirow[b]{2}{*}{$E_{\mathrm{red}}^{c}(\mathrm{~V})$} & \multirow[b]{2}{*}{ HOMO $(\mathrm{eV})$} & \multirow[b]{2}{*}{ LUMO $(\mathrm{eV})$} & \multirow[b]{2}{*}{$E_{\mathrm{g}} \mathrm{CV}^{d}(\mathrm{eV})$} & \multirow[b]{2}{*}{$\mu_{\mathrm{e}}\left(\mathrm{cm}^{2} \mathrm{~V}^{-1} \mathrm{~s}^{-1}\right)$} \\
\hline & solution & film & & & & & & & & \\
\hline F5IC & 666 & 694 & $1.9 \times 10^{5}$ & 1.64 & 1.02 & -0.75 & -5.82 & -4.05 & 1.77 & $8.1 \times 10^{-5}$ \\
\hline F7IC & 690 & 730 & $2.0 \times 10^{5}$ & 1.56 & 0.94 & -0.79 & -5.74 & -4.01 & 1.73 & $1.5 \times 10^{-4}$ \\
\hline F9IC & 710 & 746 & $2.5 \times 10^{5}$ & 1.49 & 0.72 & -0.83 & -5.52 & -3.97 & 1.55 & $1.7 \times 10^{-4}$ \\
\hline F11IC & 716 & 740 & $2.4 \times 10^{5}$ & 1.47 & 0.64 & -0.86 & -5.44 & -3.94 & 1.50 & $1.4 \times 10^{-3}$ \\
\hline
\end{tabular}

${ }^{a}$ Molar absorptivity at $\lambda_{\max }$ in solution. ${ }^{b}$ Estimated from the absorption edge in film. ${ }^{c}$ The onset oxidation and reduction potentials vs $\mathrm{FeC} \mathrm{p}_{2}{ }^{+/ 0}$. ${ }^{d} \mathrm{HOMO} / \mathrm{LUMO}$ gap from CV.

details and characterization data are presented in Supporting Information. F5IC has good solubility in common organic solvents such as chloroform and $o$-dichlorobenzene. F11IC has low solubility in chloroform (ca. $0.5 \mathrm{mg} \mathrm{mL}^{-1}$ ) and does not dissolve in $o$-dichlorobenzene at room temperature. The thermal stability was investigated using thermogravimetric analysis (TGA) and differential scanning calorimetry (DSC) (Figure S1). The decomposition temperatures $\left(T_{\mathrm{d}}, 5 \%\right.$ weight loss) of F5IC and F11IC, measured by TGA, are 344 and 400 ${ }^{\circ} \mathrm{C}$, respectively, indicating good thermal stability. The DSC 

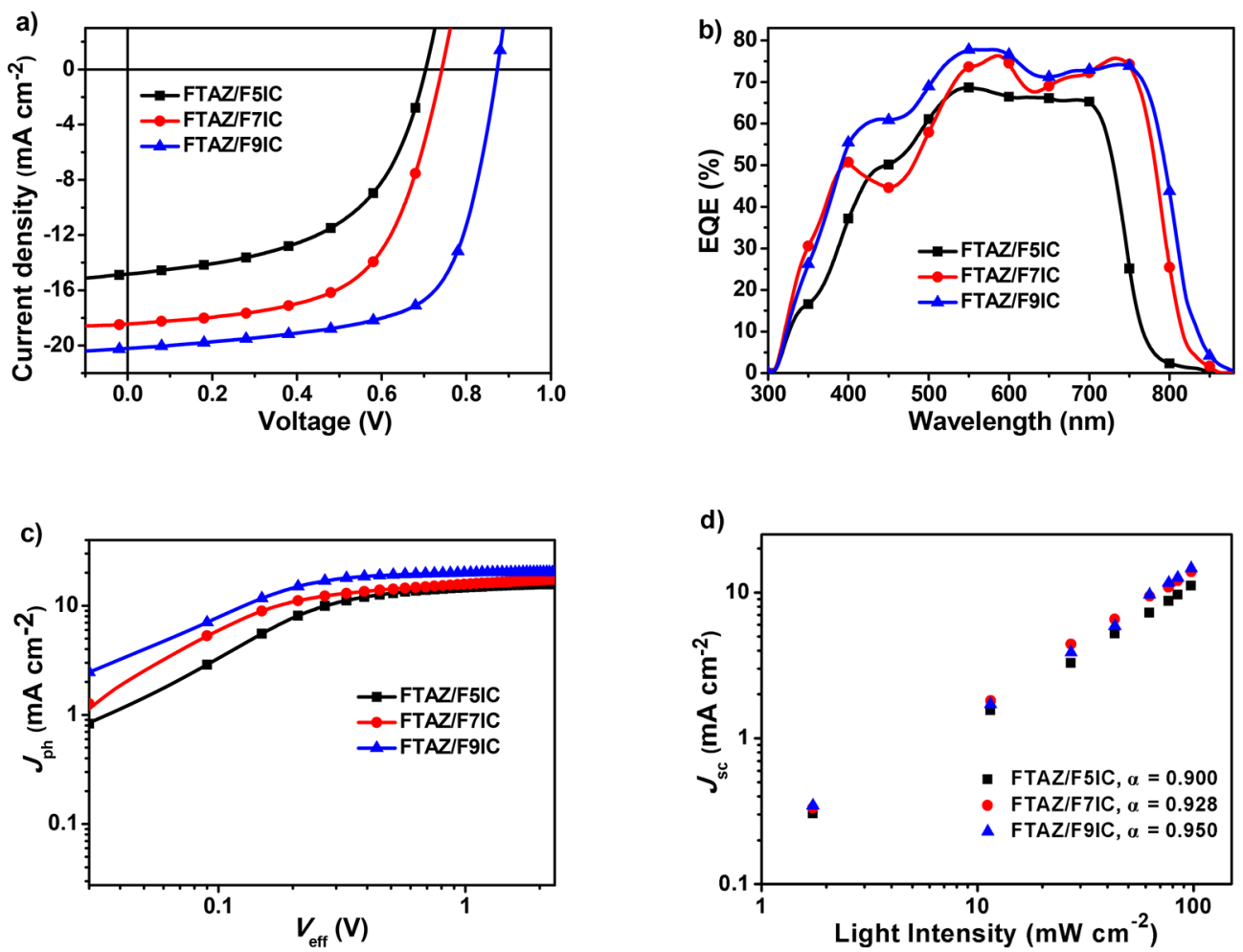

Figure 2. (a) $J-V$ characteristics (a) and EQE spectra (b) of the optimized binary-blend OSCs under illumination of an AM $1.5 \mathrm{G}$ at $100 \mathrm{~mW}$ $\mathrm{cm}^{-2}$, (c) $J_{\mathrm{ph}}$ versus $V_{\text {eff }}$ characteristics, and (d) $J_{\mathrm{SC}}$ versus light intensity of the optimized devices.

Table 2. Performance of the Optimized OSCs

\begin{tabular}{|c|c|c|c|c|c|}
\hline acceptor & $V_{\mathrm{OC}}(\mathrm{V})^{a}$ & $\left.J_{\mathrm{SC}}(\mathrm{mA} \mathrm{cm})^{-2}\right)^{a}$ & calcd $J_{S C}\left(\mathrm{~mA} \mathrm{~cm}^{-2}\right)$ & $\mathrm{FF}(\%)^{a}$ & $\operatorname{PCE}(\%)^{a}$ \\
\hline F5IC & $0.703 \pm 0.012(0.704)$ & $14.49 \pm 0.39(14.88)$ & 14.32 & $52.0 \pm 1.7(53.7)$ & $5.3 \pm 0.3(5.6)$ \\
\hline F7IC & $0.741 \pm 0.011(0.742)$ & $18.30 \pm 0.19(18.43)$ & 17.60 & $57.5 \pm 2.6(59.7)$ & $7.8 \pm 0.4(8.2)$ \\
\hline $\begin{array}{l}\text { F9IC } \\
\text { F11IC }^{b}\end{array}$ & $0.856 \pm 0.017(0.873)$ & $19.72 \pm 0.52(20.20)$ & 19.35 & $67.7 \pm 1.3(66.4)$ & $11.4 \pm 0.3(11.7)$ \\
\hline $95 \%$ F5IC + 5\% F11IC & $0.700 \pm 0.010(0.709)$ & $14.98 \pm 0.39(15.32)$ & 14.70 & $57.2 \pm 1.6(57.7)$ & $6.0 \pm 0.3(6.3)$ \\
\hline $95 \%$ F7IC+5\%F11IC & $0.744 \pm 0.012(0.747)$ & $18.61 \pm 0.18(18.77)$ & 18.16 & $61.4 \pm 2.8(63.9)$ & $8.5 \pm 0.5(9.0)$ \\
\hline $95 \% \mathrm{~F} 9 \mathrm{IC}+5 \% \mathrm{~F} 11 \mathrm{IC}$ & $0.870 \pm 0.019(0.883)$ & $20.34 \pm 0.61(20.90)$ & 20.30 & $69.9 \pm 1.9(68.0)$ & $12.4 \pm 0.2(12.6)$ \\
\hline
\end{tabular}

${ }^{a}$ Average values with standard deviation were obtained from 20 devices; the values in parentheses are the parameters of the best device. ${ }^{b}$ No entries due to limited solubility.

curves reveal that F5IC, F7IC, and F9IC have phase transitions, while F11IC shows no phase transition below $300{ }^{\circ} \mathrm{C}$.

The absorption spectra of these four FREAs were carried out in dilute chloroform solution and thin film, respectively. F5IC, F7IC, F9IC, and F11IC show gradually red-shifted absorption spectra with absorption peaks from 666 to $716 \mathrm{~nm}$ in solution (Figure 1a) as the core size increases. The maximum molar extinction coefficients are $1.9 \times 10^{5}$ to $2.5 \times 10^{5} \mathrm{M}^{-1} \mathrm{~cm}^{-1}$ (Table 1). The thin films of all four molecules show red-shifted and broadened absorption spectra with peaks at $694-746 \mathrm{~nm}$ relative to their solutions (Figure $1 \mathrm{~b}$ ). F11IC shows absorption at $550-740 \mathrm{~nm}$ stronger than that of F9IC due to the larger conjugation backbone. However, F11IC and F9IC show similar absorption peak and absorption edge, implying that further extending the core does not further red-shift the absorption spectra.

Cyclic voltammetry (CV) was used to measure the electrochemical properties of the four compounds (Figure 1c). Because each of the four compounds exhibits irreversible reduction and oxidation waves, the highest occupied molecular orbital (HOMO) and lowest unoccupied molecular orbital (LUMO) energy levels are estimated from the onset oxidation and reduction potentials, respectively, assuming the absolute energy level of $\mathrm{FeCp}_{2}{ }^{+/ 0}$ to be $4.8 \mathrm{eV}$ below vacuum. The LUMO level up-shifts from -4.05 to $-3.94 \mathrm{eV}$, while the HOMO level up-shifts from -5.82 to $-5.44 \mathrm{eV}$ (Figure 1d) as the core size extends from 5 to 11 fused rings.

The electron mobility of the four FREAs, estimated from the space charge-limited current (SCLC) method, increases from $8.1 \times 10^{-5}$ to $1.4 \times 10^{-3} \mathrm{~cm}^{2} \mathrm{~V}^{-1} \mathrm{~s}^{-1}$, as the core size extends from 5 to 11 fused rings (Table 1, Figure S2).

Photovoltaic Properties. We fabricated OSCs with a structure of indium tin oxide (ITO)/ZnO/PFN-Br/FTAZ:FREA/MoOx/Ag. FTAZ is a widely used wide-bandgap polymer donor with strong absorption at $400-620 \mathrm{~nm}$ (Figure S3) and a high hole mobility of $1.2 \times 10^{-3} \mathrm{~cm}^{2} \mathrm{~V}^{-1} \mathrm{~s}^{-1}$. 45 The absorption spectra of the donor and acceptors are complementary, and their energy levels and mobilities fit well. The optimized donor/acceptor $(D / A)$ weight ratio is $1: 1.5(\mathrm{w} / \mathrm{w})$ (Table S1); the optimized content of additive 1,8-diiodooctane (DIO) is $0.25 \%(\mathrm{v} / \mathrm{v})$ (Table S2). Figure 2a shows the current 
(a)
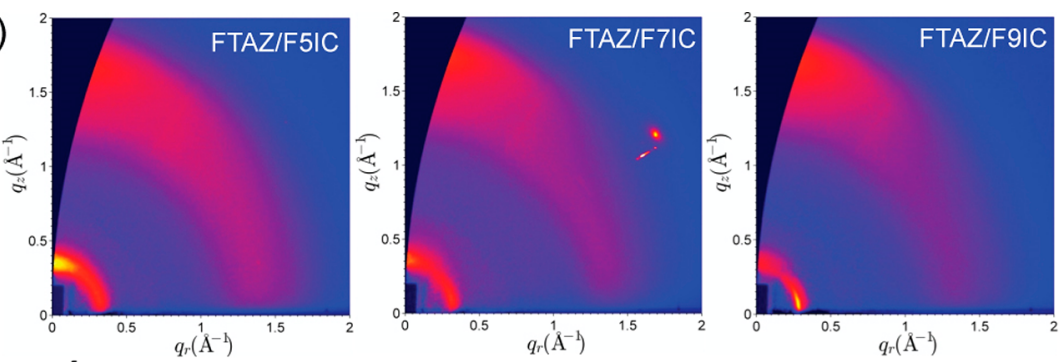

(b)

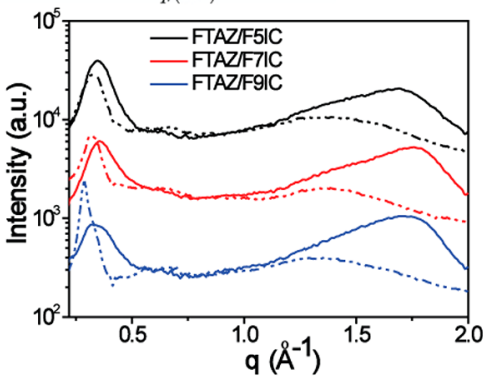

(c)

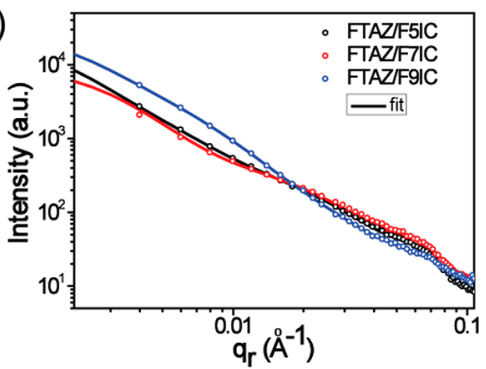

Figure 3. (a) 2D GIWAXS patterns. (b) The corresponding GIWAXS intensity profiles along the in-plane (dashed line) and out-of-plane (solid line) directions. (c) GISAXS intensity profiles and best fittings along the in-plane direction.

density-voltage $(J-V)$ curves of the best binary-blend OSCs. Due to the low solubility of F11IC, no photovoltaic effect was observed from FTAZ/F11IC-based OSCs.

The open circuit voltage $\left(V_{\mathrm{OC}}\right)$ of three binary-blend OSCs increases from 0.704 to $0.873 \mathrm{~V}$ as the aromatic core size of the acceptor extends, which is due to the upshift of LUMO levels in F5IC, F7IC, and F9IC (Table 2). The short circuit current density $\left(J_{\mathrm{SC}}\right)$ increases from 14.88 to $20.20 \mathrm{~mA} \mathrm{~cm}^{-2}$ as the core size of the acceptor extends, which is related to the redshift of absorption spectra. The fill factor (FF) increases from 53.7 to $66.4 \%$ as the core size of the acceptor extends, which benefits from enhancement of electron mobility. Finally, the best PCE increases from 5.6 to $11.7 \%$ as the core size of the acceptor extends from 5 to 9 fused rings. The photon energy loss $\left(E_{\text {loss }}\right)\left(E_{\text {loss }}=E_{\mathrm{g}}-\mathrm{e} V_{\mathrm{OC}}\right)^{46}$ of the F5IC, F7IC, and F9ICbased OSCs is $0.94,0.76$, and $0.61 \mathrm{eV}$, respectively; $E_{\text {loss }}$ decreases as the core size of the acceptor increases. The external quantum efficiency (EQE) spectra of three OSCs are broadened gradually in near-infrared wavelength from F5IC to F9IC (Figure 2b), which is consistent of the neat film absorption profile of these three acceptors (Figure 1b). The integrated $J_{\mathrm{SC}}$ of the devices calculated from the EQE spectra with the AM 1.5G reference spectrum are consistent with the $J_{\text {SC }}$ measured from $J-V$ (the error is $<5 \%$, Table 2 ).

Among the four acceptors, F11IC has the highest LUMO level, which is beneficial for achieving high $V_{\mathrm{OC}}$ in OSCs. Moreover, F11IC has the highest electron mobility, which is similar to the hole mobility of FTAZ; balanced charge mobilities of the donor and acceptor are required for high FF in devices. Although F11IC cannot be used for binaryblend devices due to its limited solubility, it can be used in ternary-blend devices as a third component if only a small amount is required. Thus, we added F11IC into FTAZ/F5IC, FTAZ/F7IC, and FTAZ/F9IC blends as a third component to fabricate ternary-blend OSCs (Figure S4a, Table 2). The optimized weight ratio of F11IC in the acceptor component is $5 \%$. When the F11IC weight ratio is increased to $10 \%$, insoluble particles can be observed in the blended film, leading to decrease in device performance (Table S3). The addition of F11IC increases the $V_{\mathrm{OC}}$ of three ternary OSCs due to the higher LUMO level of F11IC relative to other three acceptors. The $J_{\mathrm{SC}}$ and FF are also enhanced with the addition of $5 \%$ F11IC. Finally, all three ternary blends yield higher PCEs relative to their binary-blend counterparts. When the F11IC ratio is $5 \%$, the F9IC-based ternary devices yield the best PCE of $12.6 \%$, higher than those of F5IC and F7IC-based ternary devices (6.3 and 9.0\%, respectively). The maximum EQE and integrated $J_{\mathrm{SC}}$ values of F5IC, F7IC, and F9IC-based ternary devices are higher than those of binary devices (Figure S4b, Table 2).

To investigate the charge generation, dissociation, and extraction properties, we carried out measurements of photocurrent density $\left(J_{\mathrm{ph}}\right)$ versus the effective voltage $\left(V_{\text {eff }}\right)$ of OSCs (Figures $2 \mathrm{c}$ and $\mathrm{S} 4 \mathrm{c}$ ). Generally, the $J_{\mathrm{SC}} / J_{\text {sat }}$ characterizes the charge extraction under short-circuit condition when all the photogenerated excitons are dissociated into free charge carriers and collected by electrodes at high $V_{\text {eff }}$ $(>2 \mathrm{~V})$. The calculated $J_{\mathrm{SC}} / J_{\text {sat }}$ of F5IC-, F7IC-, and F9ICbased binary OSCs and F5IC/F11IC, F7IC/F11IC, and F9IC/ F11IC-based ternary OSCs are 87.4, 89.8, 94.2, 88.6, 92.3, and $95.5 \%$, respectively. It appears that the increase in core size leads to more efficient charge dissociation and collection, and the addition of F11IC further enhances the charge dissociation and collection.

Charge recombination is investigated from the $J_{\mathrm{SC}}$ versus light density $(P)$ curves, which describes as $J_{\mathrm{SC}} \propto P^{\alpha}$. ${ }^{47}$ There is only negligible charge recombination occurred in OSCs if the $\alpha$ value is 1 . The $\alpha$ values of F5IC-, F7IC-, and F9IC-based binary OSCs and F5IC/F11IC-, F7IC/F11IC-, and F9IC/ F11IC-based ternary OSCs are 0.900, 0.928, 0.950, 0.953, 0.960 , and 0.977 , respectively. Apparently, the increase in core size leads to decrease in bimolecular charge recombination, and the addition of F11IC further reduces bimolecular charge recombination (Figures $2 \mathrm{~d}$ and $\mathrm{S} 4 \mathrm{~d}$ ).

SCLC method was employed to investigate the hole and electron mobilities of the blended films (Figure S5, Table S4). For the binary blends, the hole mobilities are similar $(3.0 \times$ $10^{-4}$ to $2.1 \times 10^{-4} \mathrm{~cm}^{2} \mathrm{~V}^{-1} \mathrm{~s}^{-1}$ ), while the electron mobility increases from $3.4 \times 10^{-5}$ to $1.5 \times 10^{-4} \mathrm{~cm}^{2} \mathrm{~V}^{-1} \mathrm{~s}^{-1}$ as the acceptor core size extends from 5 to 9 fused rings, and the 
corresponding hole/electron mobility ratio $\left(\mu_{\mathrm{h}} / \mu_{\mathrm{e}}\right)$ decreases from 8.8 to 1.4. Effects of the acceptor core size on charge transport in ternary blends are similar to that in the binary blends. The addition of F11IC leads to faster and more balanced charge transport relative to the binary blends, which is beneficial for high FF.

Film Morphology. We used atomic force microscopy (AFM) to characterize the surface morphology of the four neat acceptor films, the binary and ternary blends (Figure S6). The root-mean-square roughness $\left(R_{\mathrm{q}}\right)$ of F11IC neat film is larger than that of other three acceptor neat films; $R_{\mathrm{q}}$ of FTAZ/ F11IC binary blend film is larger than that of other three acceptor binary blend films, and $R_{\mathrm{q}}$ of the ternary blends $(0.89-1.20 \mathrm{~nm})$ is larger than that of the corresponding binary blends $(0.80-0.99 \mathrm{~nm})$, which is related to the high crystallinity of F11IC.

Grazing incidence wide-angle X-ray scattering (GIWAXS) measurements were employed to investigate the film crystallinity and molecular packing information. ${ }^{48,49}$ Figures 3, S7, and S8 present the two-dimensional (2D) GIWAXS patterns and the corresponding intensity profiles of the binaryblend, ternary-blend, and pure acceptor films. The pure F5IC shows no obvious scattering peaks, indicative of its amorphous nature. The pure F7IC film shows a very weak lamellar peak along the $q_{\mathrm{r}}$ axis $\left(q=0.330 \AA^{-1}, d=19.0 \AA\right)$, demonstrating that it has weak face-on orientation. The pure F9IC film presents a notably higher crystallinity with a strong and sharp lamellar peak along the $q_{\mathrm{r}}$ axis $\left(q=0.30 \AA^{-1}, d=20.9 \AA\right)$ and a distinctive $\pi-\pi$ peak along the $q_{\mathrm{z}}$ axis $\left(q=1.77 \AA^{-1}, d=3.55\right.$ $\AA)$. The pure F11IC film exhibits the highest crystallinity compared with the other three, however, not much preferential orientation, as reflected by the appearance of several strong ring-likes scattering peaks. As the acceptor core size extends from 5 to 11 fused rings, the molecular packing is stronger, leading to higher electron mobility. When these acceptors are mixed with FTAZ, which has shown preferentially face-on orientation in its blend with other FREAs, ${ }^{43}$ quite different molecular packing behaviors are observed. For the FTAZ/ F5IC film, a strong lamellar peak appears at $q_{\mathrm{z}}=0.350 \AA^{-1}(d$ $=17.9 \AA)$, and a relatively weaker lamellar peak appears at $q_{\mathrm{r}}=$ $0.320 \AA^{-1}$. Because the lamellar peak of FTAZ was reported to appear at $q_{\mathrm{r}}=0.320 \AA^{-1,43}$ we attribute the former to the edgeon oriented F5IC domains and the latter to the face-on oriented FTAZ domains. Furthermore, a broadened $\pi-\pi$ peak appears at $q_{\mathrm{z}}=1.65 \AA^{-1}(d=3.76 \AA)$, possibly due to the faceon oriented F5IC domains or the synergistic contribution of both F5IC and FTAZ because the $\pi-\pi$ peak of FTAZ was reported to appear at $q_{\mathrm{r}}=1.7 \AA^{-1}{ }^{43}$ The scattering pattern of FTAZ/F7IC film appears to be similar to that of FTAZ/F5IC film except for a much weaker edge-on lamellar peak $\left(q_{\mathrm{z}}=\right.$ $0.340 \AA^{-1}, d=18.5 \AA$ ), and a more distinctive face-on $\pi-\pi$ peak that appeared at relatively larger $q\left(q_{\mathrm{z}}=1.67 \AA^{-1}, d=3.76\right.$ $\AA$ ). This indicates a slight enhancement of face-on ordering of the acceptor domains, consistent with the increased electron mobility in this film. Due to the strong face-on ordering of both F9IC and FTAZ, the FTAZ/F9IC film demonstrates the highest face-on ordering among the binary films with a lamellar peak at $q_{\mathrm{r}}=0.290 \AA^{-1}$ and a $\pi-\pi$ peak at $q_{\mathrm{z}}=1.70 \AA^{-1}$, originating from the face-on oriented F9IC domains and FTAZ domains, respectively. With the addition of the high-crystalline F11IC, the crystallinity and face-on ordering is further strengthened for all the ternary devices (Figures S7), consistent with the obtained higher FF and PCE.
Two-dimensional grazing incidence small-angle X-ray scattering (GISAXS) patterns and intensity profiles along inplane direction of the pure and blended films are presented in Figures $3 c$ and S9 to estimate the nanoscale phase separation information. We adopt the Debye-Anderson-Brumberger (DAB) model, a polydispersed hard sphere model, and a fractal-like network model to account for the scattering contribution from intermixing amorphous phases, FTAZ domains, and acceptor domains, respectively. ${ }^{49}$ The fitted parameters are summarized in Table S5. The correlation lengths of the intermixing phase are 49, 28, 39, 38, 40, and 21 nm for FTAZ/F5IC, FTAZ/F7IC, FTAZ/F9IC, FTAZ/F5IC/ F11IC, FTAZ/F7IC/F11IC, and FTAZ/F9IC/F11IC, respectively. All the blend films manifest a scattering shoulder at $\sim 0.07 \AA^{-1}$, which is attributed to the scattering from pure FTAZ domains of an averaged size of $\sim 9 \mathrm{~nm}$. Because the scattering of the pure F7IC film is very weak, we ignore its scattering contribution in the FTAZ/F7IC film. Although the pure F11IC film shows the strongest scattering, it contributes no distinctive scattering features in the FTAZ/F9IC/F11IC film. Therefore, we consider F9IC and F11IC indistinguishable upon GISAXS for the ternary blend film. The sizes of acceptor domains are fitted to be 73, 23, 50, 77, and $23 \mathrm{~nm}$ for FTAZ/ F5IC, FTAZ/F9IC, FTAZ/F5IC/F11IC, FTAZ/F7IC/F11IC, and FTAZ/F9IC/F11IC blend films, respectively. Compared to other films, the FTAZ/F9IC/F11IC film demonstrates relatively smaller intermixing and acceptor domains, which facilitates the exciton dissociation contributing to a higher $J_{\mathrm{SC}}$ of the devices.

\section{CONCLUSION}

These four FREAs in this study have the same end-groups and side-chains, but their cores have different sizes ranging from 5 to 11 fused rings. The core size has considerable effects on their electronic, optical, charge transport, morphological, and photovoltaic properties. Extending the acceptor core size leads to red-shift of absorption spectra; F7IC, F9IC, and F11IC films exhibit ca. $40 \mathrm{~nm}$ red-shift of absorption peaks relative to those of F5IC. However, F11IC shows an absorption profile very similar to that of F9IC, implying that further extending the core size with fused-thiophene is not able to further extend the film absorption. Increasing core size from 5 to 11 fused rings up-shifts the HOMO level from -5.82 to $-5.44 \mathrm{eV}$ and the LUMO level from -4.05 to $-3.94 \mathrm{eV}$, leading to the bandgap lowering from 1.64 to $1.47 \mathrm{eV}$. As the acceptor core size extends from 5 to 11 fused rings, the molecular packing is stronger, and the electron mobility increases from $8.1 \times 10^{-5}$ to $1.4 \times 10^{-3} \mathrm{~cm}^{2} \mathrm{~V}^{-1} \mathrm{~s}^{-1}$ in pure acceptor films. In binaryblend devices, as the acceptor core size extends from 5 to 9 fused rings, the best PCE increases from 5.6 to $11.7 \%$. This core engineering approach can simultaneously enhance $V_{\mathrm{OC}}$, $J_{\mathrm{SC}}$, and FF. $V_{\mathrm{OC}}$ increases from 0.704 to $0.873 \mathrm{~V}$ due to the upshift of LUMO level. $J_{\text {SC }}$ increases from 14.88 to $20.20 \mathrm{~mA}$ $\mathrm{cm}^{-2}$, which benefits from the red-shift of absorption spectra, more efficient charge dissociation and collection, and reduced bimolecular charge recombination. FF increases from 53.7 to $66.4 \%$, which benefits from enhancement of electron mobility, more balanced charge transport, and reduced bimolecular charge recombination.

Furthermore, we used F11IC as the third component to fabricate ternary-blend devices. As the acceptor core size extends from 5 to 9 fused rings, the best PCE increases from 6.3 to $12.6 \%$. The ternary-blend OSCs yield higher $V_{\mathrm{OC}}, J_{\mathrm{SC}}$, 
$\mathrm{FF}$, and PCE than their binary counterparts. The higher $V_{\mathrm{OC}}$ is attributed to the higher LUMO level of F11IC in comparison with F5IC, F7IC, and F9IC. The higher $J_{\mathrm{SC}}$ is related to more efficient charge dissociation and collection and reduced bimolecular charge recombination. The higher FF is due to higher electron mobility, more balanced charge transport, and reduced bimolecular charge recombination.

\section{ASSOCIATED CONTENT}

\section{S Supporting Information}

The Supporting Information is available free of charge on the ACS Publications website at DOI: 10.1021/acs.chemmater.8b02222.

Materials synthesis and characterization; TGA and DSC curves, SCLC, AFM images, GIWAXS, and GISAXS data; device fabrication, optimization, and characterization (PDF)

\section{AUTHOR INFORMATION}

\section{Corresponding Author}

*E-mail: xwzhan@pku.edu.cn.

\section{ORCID}

Xiaowei Zhan: 0000-0002-1006-3342

\section{Notes}

The authors declare no competing financial interest.

\section{ACKNOWLEDGMENTS}

X.Z. thank NSFC (Grants 21734001 and 51761165023). X.L. thanks the financial support from NSFC/RGC Joint Research Scheme (Grant N_CUHK418/17), Research Grant Council of Hong Kong (General Research Fund 14314216 and Themebased Research Scheme T23-407/13-N), the beam time and technical supports provided by 23A SWAXS beamline at NSRRC, Hsinchuand BL19 beamline at SSRF, Shanghai. J.J.R. and W.Y. were supported by the National Science Foundation (Grant CBET-1639429).

\section{REFERENCES}

(1) Li, G.; Chang, W. H.; Yang, Y. Low-bandgap conjugated polymers enabling solution-processable tandem solar cells. Nat. Rev. Mater. 2017, 2, 17043.

(2) Lu, L. Y.; Kelly, M. A.; You, W.; Yu, L. P. Status and prospects for ternary organic photovoltaics. Nat. Photonics 2015, 9, 491-500.

(3) Lin, Y.; Li, Y.; Zhan, X. Small molecule semiconductors for highefficiency organic photovoltaics. Chem. Soc. Rev. 2012, 41, 42454272.

(4) Zhao, J.; Li, Y.; Yang, G.; Jiang, K.; Lin, H.; Ade, H.; Ma, W.; Yan, H. Efficient organic solar cells processed from hydrocarbon solvents. Nat. Energy 2016, 1, 15027.

(5) Li, M.; Gao, K.; Wan, X.; Zhang, Q.; Kan, B.; Xia, R.; Liu, F.; Yang, X.; Feng, H.; Ni, W.; Wang, Y.; Peng, J.; Zhang, H.; Liang, Z.; Yip, H.-L.; Peng, X.; Cao, Y.; Chen, Y. Solution-processed organic tandem solar cells with power conversion efficiencies $>12 \%$. Nat. Photonics 2017, 11, 85-90.

(6) Zhang, S.; Qin, Y.; Zhu, J.; Hou, J. Over 14\% Efficiency in Polymer Solar Cells Enabled by a Chlorinated Polymer Donor. Adv. Mater. 2018, 30, 1800868.

(7) Xu, X.; Yu, T.; Bi, Z.; Ma, W.; Li, Y.; Peng, Q. Realizing Over 13\% Efficiency in Green-Solvent-Processed Nonfullerene Organic Solar Cells Enabled by 1,3,4-Thiadiazole-Based Wide-Bandgap Copolymers. Adv. Mater. 2018, 30, 1703973.

(8) Fei, Z.; Eisner, F. D.; Jiao, X.; Azzouzi, M.; Rohr, J. A.; Han, Y.; Shahid, M.; Chesman, A. S. R.; Easton, C. D.; McNeill, C. R.; Anthopoulos, T. D.; Nelson, J.; Heeney, M. An Alkylated
Indacenodithieno[3,2-b]thiophene-Based Nonfullerene Acceptor with High Crystallinity Exhibiting Single Junction Solar Cell Efficiencies Greater than $13 \%$ with Low Voltage Losses. Adv. Mater. 2018, 30, 1705209.

(9) Chen, J. D.; Li, Y. Q.; Zhu, J.; Zhang, Q.; Xu, R. P.; Li, C.; Zhang, Y. X.; Huang, J. S.; Zhan, X.; You, W.; Tang, J. X. Polymer Solar Cells with 90\% External Quantum Efficiency Featuring an Ideal Light- and Charge-Manipulation Layer. Adv. Mater. 2018, 30, 1706083.

(10) Lin, Y.; Zhan, X. Oligomer Molecules for Efficient Organic Photovoltaics. Acc. Chem. Res. 2016, 49, 175-183.

(11) Hou, J.; Inganäs, O.; Friend, R. H.; Gao, F. Organic solar cells based on non-fullerene acceptors. Nat. Mater. 2018, 17, 119.

(12) Yan, C.; Barlow, S.; Wang, Z.; Yan, H.; Jen, A. K. Y.; Marder, S. R.; Zhan, X. Non-fullerene acceptors for organic solar cells. Nat. Rev. Mater. 2018, 3, 18003.

(13) Cheng, P.; Li, G.; Zhan, X.; Yang, Y. Next-generation organic photovoltaics based on non-fullerene acceptors. Nat. Photonics 2018, $12,131-142$

(14) Zhan, X.; Facchetti, A.; Barlow, S.; Marks, T. J.; Ratner, M. A.; Wasielewski, M. R.; Marder, S. R. Rylene and Related Diimides for Organic Electronics. Adv. Mater. 2011, 23, 268-284.

(15) Zhang, J.; Li, Y.; Huang, J.; Hu, H.; Zhang, G.; Ma, T.; Chow, P. C. Y.; Ade, H.; Pan, D.; Yan, H. Ring-Fusion of Perylene Diimide Acceptor Enabling Efficient Nonfullerene Organic Solar Cells with a Small Voltage Loss. J. Am. Chem. Soc. 2017, 139, 16092-16095.

(16) Meng, D.; Fu, H.; Xiao, C.; Meng, X.; Winands, T.; Ma, W.; Wei, W.; Fan, B.; Huo, L.; Doltsinis, N. L.; Li, Y.; Sun, Y.; Wang, Z. Three-Bladed Rylene Propellers with Three-Dimensional Network Assembly for Organic Electronics. J. Am. Chem. Soc. 2016, 138, 10184-10190.

(17) Wu, Q.; Zhao, D.; Schneider, A. M.; Chen, W.; Yu, L. Covalently Bound Clusters of Alpha-Substituted PDI-Rival Electron Acceptors to Fullerene for Organic Solar Cells. J. Am. Chem. Soc. 2016, 138, 7248-7251.

(18) Zhong, Y.; Trinh, M. T.; Chen, R.; Purdum, G. E.; Khlyabich, P. P.; Sezen, M.; Oh, S.; Zhu, H.; Fowler, B.; Zhang, B.; Wang, W.; Nam, C. Y.; Sfeir, M. Y.; Black, C. T.; Steigerwald, M. L.; Loo, Y. L.; Ng, F.; Zhu, X. Y.; Nuckolls, C. Molecular helices as electron acceptors in high-performance bulk heterojunction solar cells. Nat. Commun. 2015, 6, 8242

(19) Li, H.; Earmme, T.; Ren, G.; Saeki, A.; Yoshikawa, S.; Murari, N. M.; Subramaniyan, S.; Crane, M. J.; Seki, S.; Jenekhe, S. A. Beyond fullerenes: design of nonfullerene acceptors for efficient organic photovoltaics. J. Am. Chem. Soc. 2014, 136, 14589-14597.

(20) Zhang, X.; Lu, Z.; Ye, L.; Zhan, C.; Hou, J.; Zhang, S.; Jiang, B.; Zhao, Y.; Huang, J.; Zhang, S.; Liu, Y.; Shi, Q.; Liu, Y.; Yao, J. A Potential Perylene Diimide Dimer-Based Acceptor Material for Highly Efficient Solution-Processed Non-Fullerene Organic Solar Cells with 4.03\% Efficiency. Adv. Mater. 2013, 25, 5791.

(21) Zhan, X.; Tan, Z. a.; Domercq, B.; An, Z.; Zhang, X.; Barlow, S.; Li, Y.; Zhu, D.; Kippelen, B.; Marder, S. R. A high-mobility electron-transport polymer with broad absorption and its use in fieldeffect transistors and all-polymer solar cells. J. Am. Chem. Soc. 2007, $129,7246-7247$.

(22) Eastham, N. D.; Dudnik, A. S.; Aldrich, T. J.; Manley, E. F.; Fauvell, T. J.; Hartnett, P. E.; Wasielewski, M. R.; Chen, L. X.; Melkonyan, F. S.; Facchetti, A.; Chang, R. P. H.; Marks, T. J. Small Molecule Acceptor and Polymer Donor Crystallinity and Aggregation Effects on Microstructure Templating: Understanding Photovoltaic Response in Fullerene-Free Solar Cells. Chem. Mater. 2017, 29, 4432-4444.

(23) Aldrich, T. J.; Swick, S. M.; Melkonyan, F. S.; Marks, T. J. Enhancing Indacenodithiophene Acceptor Crystallinity via Substituent Manipulation Increases Organic Solar Cell Efficiency. Chem. Mater. 2017, 29, 10294-10298.

(24) Eastham, N. D.; Logsdon, J. L.; Manley, E. F.; Aldrich, T. J.; Leonardi, M. J.; Wang, G.; Powers-Riggs, N. E.; Young, R. M.; Chen, L. X.; Wasielewski, M. R.; Melkonyan, F. S.; Chang, R. P. H.; Marks, 
T. J. Hole-Transfer Dependence on Blend Morphology and Energy Level Alignment in Polymer: ITIC Photovoltaic Materials. Adv. Mater. 2018, 30, 1704263.

(25) Lin, Y.; Wang, J.; Zhang, Z.-G.; Bai, H.; Li, Y.; Zhu, D.; Zhan, $X$. An Electron Acceptor Challenging Fullerenes for Efficient Polymer Solar Cells. Adv. Mater. 2015, 27, 1170-1174.

(26) Xu, S. J.; Zhou, Z.; Liu, W.; Zhang, Z.; Liu, F.; Yan, H.; Zhu, X. A Twisted Thieno[3,4-b]thiophene-Based Electron Acceptor Featuring a 14-pi-Electron Indenoindene Core for High-Performance Organic Photovoltaics. Adv. Mater. 2017, 29, 1704510.

(27) Lin, Y.; He, Q.; Zhao, F.; Huo, L.; Mai, J.; Lu, X.; Su, C. J.; Li, T.; Wang, J.; Zhu, J.; Sun, Y.; Wang, C.; Zhan, X. A Facile Planar Fused-Ring Electron Acceptor for As-Cast Polymer Solar Cells with 8.71\% Efficiency. J. Am. Chem. Soc. 2016, 138, 2973-2976.

(28) Feng, S.; Zhang, C.; Liu, Y.; Bi, Z.; Zhang, Z.; Xu, X.; Ma, W.; Bo, Z. Fused-Ring Acceptors with Asymmetric Side Chains for HighPerformance Thick-Film Organic Solar Cells. Adv. Mater. 2017, 29, 1703527.

(29) Baran, D.; Ashraf, R. S.; Hanifi, D. A.; Abdelsamie, M.; Gasparini, N.; Rohr, J. A.; Holliday, S.; Wadsworth, A.; Lockett, S.; Neophytou, M.; Emmott, C. J.; Nelson, J.; Brabec, C. J.; Amassian, A.; Salleo, A.; Kirchartz, T.; Durrant, J. R.; McCulloch, I. Reducing the efficiency-stability-cost gap of organic photovoltaics with highly efficient and stable small molecule acceptor ternary solar cells. Nat. Mater. 2017, 16, 363-369.

(30) Dai, S.; Li, T.; Wang, W.; Xiao, Y.; Lau, T. K.; Li, Z.; Liu, K.; $\mathrm{Lu}, \mathrm{X}$.; Zhan, X. Enhancing the Performance of Polymer Solar Cells via Core Engineering of NIR-Absorbing Electron Acceptors. Adv. Mater. 2018, 30, 1706571.

(31) Wang, W.; Yan, C.; Lau, T. K.; Wang, J.; Liu, K.; Fan, Y.; Lu, X.; Zhan, X. Fused Hexacyclic Nonfullerene Acceptor with Strong Near-Infrared Absorption for Semitransparent Organic Solar Cells with 9.77\% Efficiency. Adv. Mater. 2017, 29, 1701308.

(32) Zhang, J. X.; Yan, C. Q.; Wang, W.; Xiao, Y. Q.; Lu, X. H.; Barlow, S.; Parker, T. C.; Zhan, X. W.; Marder, S. R. Panchromatic Ternary Photovoltaic Cells Using a Nonfullerene Acceptor Synthesized Using C-H Functionalization. Chem. Mater. 2018, 30, 309-313.

(33) Lin, Y.; Zhao, F.; He, Q.; Huo, L.; Wu, Y.; Parker, T. C.; Ma, W.; Sun, Y.; Wang, C.; Zhu, D.; Heeger, A. J.; Marder, S. R.; Zhan, X. High-Performance Electron Acceptor with Thienyl Side Chains for Organic Photovoltaics. J. Am. Chem. Soc. 2016, 138, 4955-4961.

(34) Zhao, F.; Dai, S.; Wu, Y.; Zhang, Q.; Wang, J.; Jiang, L.; Ling, Q.; Wei, Z.; Ma, W.; You, W.; Wang, C.; Zhan, X. Single-Junction Binary-Blend Nonfullerene Polymer Solar Cells with $12.1 \%$ Efficiency. Adv. Mater. 2017, 29, 1700144.

(35) Kan, B.; Zhang, J.; Liu, F.; Wan, X.; Li, C.; Ke, X.; Wang, Y.; Feng, H.; Zhang, Y.; Long, G.; Friend, R. H.; Bakulin, A. A.; Chen, Y. Fine-Tuning the Energy Levels of a Nonfullerene Small-Molecule Acceptor to Achieve a High Short-Circuit Current and a Power Conversion Efficiency over $12 \%$ in Organic Solar Cells. Adv. Mater. 2018, 30, 1704904.

(36) Li, S.; Ye, L.; Zhao, W.; Zhang, S.; Mukherjee, S.; Ade, H.; Hou, J. Energy-Level Modulation of Small-Molecule Electron Acceptors to Achieve over 12\% Efficiency in Polymer Solar Cells. Adv. Mater. 2016, 28, 9423-9429.

(37) Yang, Y.; Zhang, Z. G.; Bin, H.; Chen, S.; Gao, L.; Xue, L.; Yang, C.; Li, Y. Side-Chain Isomerization on an n-type Organic Semiconductor ITIC Acceptor Makes 11.77\% High Efficiency Polymer Solar Cells. J. Am. Chem. Soc. 2016, 138, 15011-15018.

(38) Wang, J.; Wang, W.; Wang, X.; Wu, Y.; Zhang, Q.; Yan, C.; Ma, W.; You, W.; Zhan, X. Enhancing Performance of Nonfullerene Acceptors via Side-Chain Conjugation Strategy. Adv. Mater. 2017, 29, 1702125.

(39) Li, Y.; Lin, J. D.; Che, X.; Qu, Y.; Liu, F.; Liao, L. S.; Forrest, S. R. High Efficiency Near-Infrared and Semitransparent Non-Fullerene Acceptor Organic Photovoltaic Cells. J. Am. Chem. Soc. 2017, 139, 17114-17119.
(40) Jia, B.; Dai, S.; Ke, Z.; Yan, C.; Ma, W.; Zhan, X. Breaking 10\% Efficiency in Semitransparent Solar Cells with Fused-Undecacyclic Electron Acceptor. Chem. Mater. 2018, 30, 239-245.

(41) Li, T.; Dai, S.; Ke, Z.; Yang, L.; Wang, J.; Yan, C.; Ma, W.; Zhan, X. Fused Tris(thienothiophene)-Based Electron Acceptor with Strong Near-Infrared Absorption for High-Performance As-Cast Solar Cells. Adv. Mater. 2018, 30, 1705969.

(42) Zhu, J.; Ke, Z.; Zhang, Q.; Wang, J.; Dai, S.; Wu, Y.; Xu, Y.; Lin, Y.; Ma, W.; You, W.; Zhan, X. Naphthodithiophene-Based Nonfullerene Acceptor for High-Performance Organic Photovoltaics: Effect of Extended Conjugation. Adv. Mater. 2018, 30, 1704713.

(43) Dai, S.; Zhao, F.; Zhang, Q.; Lau, T. K.; Li, T.; Liu, K.; Ling, Q.; Wang, C.; Lu, X.; You, W.; Zhan, X. Fused Nonacyclic Electron Acceptors for Efficient Polymer Solar Cells. J. Am. Chem. Soc. 2017, $139,1336-1343$.

(44) Yao, Z.; Liao, X.; Gao, K.; Lin, F.; Xu, X.; Shi, X.; Zuo, L.; Liu, F.; Chen, Y.; Jen, A. K. Dithienopicenocarbazole-Based Acceptors for Efficient Organic Solar Cells with Optoelectronic Response Over $1000 \mathrm{~nm}$ and an Extremely Low Energy Loss. J. Am. Chem. Soc. 2018, 140, 2054-2057.

(45) Li, W.; Albrecht, S.; Yang, L.; Roland, S.; Tumbleston, J. R.; McAfee, T.; Yan, L.; Kelly, M. A.; Ade, H.; Neher, D.; You, W. Mobility-controlled performance of thick solar cells based on fluorinated copolymers. J. Am. Chem. Soc. 2014, 136, 15566-15576.

(46) Veldman, D.; Meskers, S. C. J.; Janssen, R. A. J. The Energy of Charge-Transfer States in Electron Donor-Acceptor Blends: Insight into the Energy Losses in Organic Solar Cells. Adv. Funct. Mater. 2009, 19, 1939-1948.

(47) Schilinsky, P.; Waldauf, C.; Brabec, C. J. Recombination and loss analysis in polythiophene based bulk heterojunction photodetectors. Appl. Phys. Lett. 2002, 81, 3885-3887.

(48) Mai, J.; Lau, T.-K.; Li, J.; Peng, S.-H.; Hsu, C.-S.; Jeng, U. S.; Zeng, J.; Zhao, N.; Xiao, X.; Lu, X. Understanding Morphology Compatibility for High-Performance Ternary Organic Solar Cells. Chem. Mater. 2016, 28, 6186-6195.

(49) Mai, J.; Lau, T.-K.; Xiao, T.; Su, C.-J.; Jeng, U. S.; Zhao, N.; Xiao, X.; Lu, X. A ternary morphology facilitated thick-film organic solar cell. RSC Adv. 2015, 5, 88500-88507. 